\title{
Characteristics of Helicobacter pylori-seropositive subjects according to the stool antigen test findings: a prospective study
}

\author{
Hee-Won Moon ${ }^{1}$, Sun-Young Lee ${ }^{2}$, Mina Hur ${ }^{1}$, and Yeo-Min Yun ${ }^{1}$
}

Departments of ${ }^{1}$ Laboratory Medicine and ${ }^{2}$ Internal Medicine, Konkuk University School of Medicine, Seoul, Korea

Received: November 10, 2016 Revised : January 15, 2017

Accepted: February 11, 2017

\section{Correspondence to}

Sun-Young Lee, M.D.

Department of Internal Medicine, Konkuk University School of Medicine, 120-1 Neungdong-ro, Gwangjin-gu, Seoul 05030, Korea Tel: +82-2-2030-7747

Fax: +82-2-2030-7748

E-mail: sunyoung@kuh.ac.kr
Background/Aims: In countries with a higher risk of gastric atrophic gastritis, noninvasive tests are helpful for a more reliable diagnosis of Helicobacter pylori infection. The aim of this study was to evaluate the characteristics of seropositive subjects according to their stool $\mathrm{H}$. pylori antigen test, serum pepsinogen (PG) assay, and endoscopic findings.

Methods: Consecutive subjects who visited Konkuk University Medical Center for upper gastrointestinal endoscopy for a regular check-up were included in a prospective setting if the serum anti-H. pylori immunoglobulin $\mathrm{G}$ assay was positive. A H. pylori antigen stool test was measured using a stool $H$. pylori antigen enzyme-linked immunosorbent assay kit on the same day as a serum PG assay and endoscopy.

Results: Of 318 seropositive subjects, 256 (80.5\%) showed positive stool test findings. Subjects with a negative stool test result showed lower serum PG I $(p<0.001)$ and PG II $(p<0.001)$ levels and higher PG I/II ratio $(p<0.001)$ than those with a positive stool test. Chronic atrophic gastritis was more common in the positive stool test group than the negative stool test group on endoscopic finding $(p=$ 0.009). A higher serum PG I level $(p=0.001)$ and a lower serum PG I/II ratio ( $p=$ $0.001)$ were independent risk factors for the presence of $H$. pylori antigen in stool. Conclusions: A high serum PG level denotes an ongoing current $H$. pylori infection with positive stool $\mathrm{H}$. pylori antigen test findings. Seropositive subjects with increased gastric secreting ability tend to have $H$. pylori in their fecal material as reflected by a positive stool $\mathrm{H}$. pylori antigen test finding.

Keywords: Helicobacter pylori; Serology; Stool; Pepsinogen; Endoscopy

\section{INTRODUCTION}

Helicobacter pylori infection can be diagnosed by invasive methods using the gastric biopsied specimens or noninvasively by examining serum, urine, breath, or stool. Histological examination by Giemsa staining is usually recommended for the diagnosis, but it is difficult to visualize the bacterium when there is a low density of
H. pylori on the gastric mucosa with atrophic and metaplastic changes [1]. Limitations of histological examination include increased false negative test findings due to various density of $H$. pylori at different sites, high cost, long turnaround time, and interobserver variability [2]. Therefore, in East Asian countries with a higher risk of chronic atrophic gastritis (CAG), intestinal metaplasia, and gastric cancer, noninvasive tests need to be added 
for a more reliable diagnosis of $H$. pylori infection.

The monoclonal-type $H$. pylori stool antigen test is a reliable test with high specificity and sensitivity [3]. It detects the presence of current $H$. pylori infection, and is therefore useful for evaluating the status of $H$. pylori infection after eradication $[4,5]$. It is useful even in the presence of severe gastric atrophy or intestinal metaplasia, and has the potential for quantitative evaluation of the intragastric $H$. pylori status $[6,7]$. The sensitivity, specificity, and accuracy of the stool test is high, with reported rates of $93.1 \%, 94.6 \%$, and $93.8 \%$, respectively [6]. Nonetheless, the diagnostic accuracy of the H. pylori stool antigen test can be diminished in special settings, such as the presence of upper gastrointestinal bleeding, and a small percentage of antigen-positive stools may present with other Helicobacter species [8,9]. Moreover, there is a risk of a false negative result in cases in which eradication fails, if the test is performed within 2 weeks of eradication or with recent proton pump inhibitor intake therapy $[7,9]$. Nevertheless, it is still uncertain whether the stool antigen test findings differ according to the degree of gastric corpus atrophy.

The serum pepsinogen (PG) assay enables the detection of the progression to advanced gastric corpus atrophy by virtue of the stepwise reduction in the PG I level and PG I/II ratio [10,11]. In endemic area of $H$. pylori-infection, serum PG assay is widely used to predict the presence of gastric corpus atrophy [12,13]. Gastric corpus atrophy, so-called serologic gastric atrophy, is diagnosed when the serum PG I level is of $<70 \mathrm{ng} / \mathrm{mL}$ and the serum PG I/II ratio is of $<3.0[10,13]$. Most of the subjects with closed-type CAG do not have gastric corpus atrophy because their PG I-secreting cells located on the fundus are still intact $[14,15]$. Moreover, only some of the subjects with open-type CAG have gastric corpus atrophy because both the serum PG I and PG II levels increase if there is still an active $H$. pylori infection inside the stomach. Gastric corpus atrophy does not occur unless there is a large amount of mucosal cell damage on the fundus, and therefore, the prevalence of gastric corpus atrophy is lower than the prevalence of CAG. Nonetheless, little is known about the link between the stool test findings and serum PG level in seroprevalent population. The aim of this study was to determine the characteristics of seropositive subjects according to their stool $H$. pylori antigen test findings, serum PG as- says, and endoscopic diagnoses.

\section{METHODS}

\section{Subjects and study design}

Consecutive adults who visited Konkuk University Medical Center for upper gastrointestinal endoscopy for a regular check-up were included in a prospective setting from 2014 to 2015 if the serum anti-H. pylori immunoglobulin $\mathrm{G}(\mathrm{IgG})$ test was positive. Subjects were excluded if the age was below 20 years or if there was a recent use of medication. Those with a history of $\mathrm{H}$. pylori eradication or gastrectomy were also excluded. Subjects were not enrolled from the beginning when there was any possibility of organic disease, such as gastrointestinal bleeding, malignancy, acute infection, or other significant systemic disease, that required treatment. This study was approved by the Institutional Review Board of the Konkuk University Medical Center (No. KUH 1010575) and was registered as ClinicalTrials.gov (ID: KCTooo1149, https://cris.nih.go.kr). It was performed in accordance with the principles of the Declaration of Helsinki and written informed consents were obtained.

\section{Definition for $H$. pylori infection}

IgG titer was measured using the Chorus H. pylori IgG assay (DIESSE Diagnostica Senese, Monteriggioni, Italy), which has a high concurrence rate in Korean adults with the Genedia H. pylori enzyme-linked immunosorbent assay (ELISA; Green Cross Medical Science, Seoul, Korea) and the Vidas H. pylori IgG assays (BioMerieux, Marcy l'Etoile, France) [16]. The value varied from o to $200 \mathrm{AU} / \mathrm{mL}$, and was expressed as $>200 \mathrm{AU} / \mathrm{mL}$ if the value exceeded the upper limit. The subjects were defined as having infection if more than two $H$. pylori test findings were positive in the serum anti-H. pylori IgG test, $H$. pylori stool antigen test, and gastric biopsy performed on the same day.

\section{H. pylori stool antigen test}

Fresh stool samples were collected on the same day the serum anti-H. pylori antibody test was performed, and were stored at $-70^{\circ} \mathrm{C}$ for analysis. $\mathrm{H}$. pylori antigen was analyzed using the SD H. pylori antigen ELISA kit (Standard Diagnostics Inc., Yongin, Korea) according to the 
manufacturer's instructions. Briefly, the assay utilizes a mouse anti-H. pylori monoclonal antibody that is adsorbed to microplate wells. Fecal samples were diluted with diluent, and were added with controls and a peroxidase-conjugated monoclonal antibody. After the addition, incubated for an hour at $37^{\circ} \mathrm{C}$. Washing was done to remove unbound materials. After washing, substrate was added and sent for 10 minutes of incubation. The results were read using a spectrophotometer at $450 \mathrm{~nm}$. Negative result was given when the optical density (OD) of stool $H$. pylori antigen was $\leq$ the negative mean OD +o.1. Positive result was subclassified into two findings. Strongly positive result was given when OD of stool $H$. pylori antigen was $\geq 3.0$, and a weakly positive result was given when $\mathrm{OD}$ was between the mean negative OD +0.1 and 3.0.

\section{Endoscopic examination}

Upper gastrointestinal endoscopy was performed at our center using a model GIF-H26o endoscope (Olympus, Tokyo, Japan). CAG was defined as transparent visible vessels, and metaplastic gastritis (MG) was defined as whitish patches or plaques. Nodular gastritis (NG) was defined as multiple, regular, and tiny (2 to $3 \mathrm{~mm}$ )-sized nodules on the antrum without color change. Erosive gastritis was defined as multiple hyperemic, raised lesions on the antrum, while chronic superficial gastritis (CSG) was defined as several hyperemic, linear streaks mainly on the greater curvature side of the antrum. Peptic ulcer disease (PUD) was defined as a deep mucosal defect suspicious for submucosal invasion. For esophageal lesions, erosive esophagitis (EE) was diagnosed when hyperemic streak(s) were found on the lower esophagus. Endoscopic findings were initially described, and one or two biopsies were done at the lesion(s) with color and/or structural changes. The biopsied specimens were fixed in 10\% neutral buffered formalin. They were embedded in paraffin blocks thereafter, and were sectioned for Giemsa staining and Hematoxylin \& Eosin staining.

\section{Serum PG assay}

After centrifuging the fasting serum samples, PG levels were determined by a latex-enhanced turbidimetric immunoassay (HBi Co., Anyang, Korea) as described in our previous study [13]. Normal ranges for serum PG I level was defined as $>70 \mathrm{ng} / \mathrm{mL}$, PG II level as $<15 \mathrm{ng} / \mathrm{mL}$, and
PG I/II ratio as $>3.0$ as described in previous studies.

\section{Statistical analyses}

Sample size calculation was done using one correlation power analysis with a $5 \%$ significance level and a statistical power of $80 \%$. Numeric result was calculated based on our pilot data using Ha which was between Ro (the value of the population correlation under the null hypothesis) and $\mathrm{R} 1$ (the value of the population correlation under the alternative hypothesis). A decision was made to enroll more than $123 \mathrm{H}$. pylori-infected subjects (power $=0.08022, \alpha=0.0500, \beta=0.1978$, Ro $=0.0000, R_{1}=$ $0.2500)$.

Using PASW version 17.0 (SPSS Inc., Chicago, IL, USA), significance was considered at a $p$ value $<0.05$. Categorical variables including gender, endoscopic disgnoses, and stool antigen test findings are shown in frequency (\%) using the chi-square test. Continuous variables including age and serum PG levels are shown as mean value \pm standard deviation using the Student $t$ test. OD values for the stool test findings are shown as median value with ranges (minimum and maximum values) using the Kruskal-Wallis test due to its skewed distributions. A receiver operating characteristic (ROC) curve was constructed by plotting sensitivity (true-positive rate) against 1-specificity (false-positive rate) over all possible threshold levels of serum PG I, PG II, and PG I/ II ratio, which are related to the positive stool test findings. Logistic regression analysis was performed to verify the independent significance of variables correlated with the positive stool test findings using the odds ratio. Differences in 95\% confidence interval (CI) between the groups were compared additionally. For the continuous values (age and serum PG assays), unstandardized coefficients are shown with standard error using linear logistic regression analysis.

\section{RESULTS}

\section{Characteristics of the subjects}

A total of $322 \mathrm{H}$. pylori-seropositive subjects were included in the study. Four subjects were excluded due to the poor quantity of the fecal specimens (Fig. 1). Of the 318 included subjects, stool test was positive in 256 (80.5\%). 


\section{Distribution by $\mathrm{H}$. pylori stool antigen test findings}

There were no differences in age and gender between the negative and positive stool test groups. Subjects with a negative stool test result displayed significantly lower serum PG I $(p<0.001)$ and PG II $(p<0.001)$ levels than those with the positive stool test (Table 1 ). As a conse-

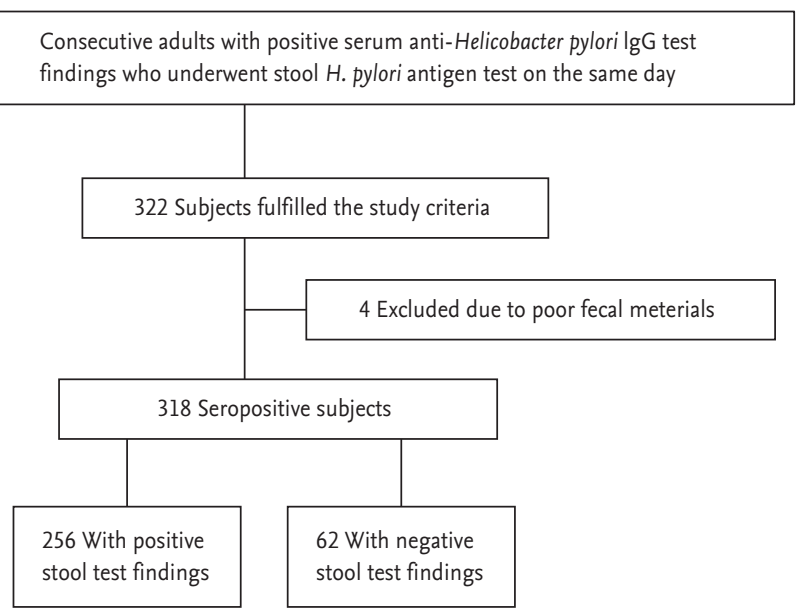

Figure 1. Study flow of the included subjects. Three hundred and twenty-two subjects with serum anti-Helicobacter pylori immunoglobulin $\mathrm{G}(\mathrm{IgG})$ level $\geq 12.0 \mathrm{AU} / \mathrm{mL}$ were included in the study. $H$. pylori antigen was not found in stool in 62 seropositive subjects. quence, serum PG I/II ratio was higher in the negative stool test group than the positive stool test group $(p<$ 0.001).

With regard to the endoscopic findings, CAG was more common in those with a positive stool test $(p=0.009)$ and, CSG was more common in those with a negative stool test $(p=0.013)$. Logistic regression analysis revealed that none of the endoscopic diagnosis was independently related to a positive stool test finding (Table 2).

\section{Link between the serum PG assay and stool antigen test findings}

On the multivariate analysis findings, serum PG I level was correlated with a positive stool test $(p=0.001)$, whereas serum PG I/II ratio was inversely related to a positive stool test $(p=0.001)$. Using the continuous values, a positive correlation was found between the stool antigen test and serum PG I $(r=0.269, p<0.001)$ and PG II $(r=0.407, p<0.001)$ titers. The stool test and serum PG I/II ratio were inversely correlated $(r=-0.355, p<0.001)$. ROC curve analyses revealed optimal cut-off values for a positive stool test finding were a serum PG I level of 41.5 $\mathrm{ng} / \mathrm{mL}$ (area under the curve [AUC], 0.723; 95\% CI, 0.636 to 0.809 , $p<0.001$ ), serum PG II level of $8.7 \mathrm{ng} / \mathrm{mL}$ (AUC, $0.750 ; 95 \% \mathrm{CI}, 0.663$ to $0.838 ; p<0.001)$, and PG I/II ratio of 3.35 (AUC, $0.698 ; 95 \%$ CI, 0.598 to $0.798 ; p<0.001$ ).

Table 1. Characteristics of the $\mathbf{3 1} 8$ Helicobacter pylori-seropositive subjects

\begin{tabular}{|c|c|c|c|c|}
\hline \multirow{2}{*}{ Variable } & \multirow{2}{*}{ All subjects $(n=318)$} & \multicolumn{3}{|c|}{ H. pylori stool antigen test } \\
\hline & & Negative $(n=62)$ & Positive $(n=256)$ & $p$ value \\
\hline Age, yr & $51.5 \pm 11.3$ & $52.2 \pm 11.1$ & $51.3 \pm 11.4$ & 0.589 \\
\hline Male sex & $182(57.2)$ & $41(66.1)$ & $141(55 \cdot 1)$ & 0.115 \\
\hline PG I, ng/mL & $63.0 \pm 28.3$ & $49.6 \pm 27.2$ & $66.3 \pm 27.6$ & $<0.001$ \\
\hline PG II, ng/mL & $18.9 \pm 10.9$ & $12.4 \pm 7.8$ & $20.5 \pm 11.0$ & $<0.001$ \\
\hline PG I/II & $4.00 \pm 1.95$ & $4.95 \pm 2.32$ & $3.76 \pm 1.78$ & $<0.001$ \\
\hline Stool H. pylori antigen test (OD) & $1.82(0.05-4.00)$ & $0.71(0.05-0.15)$ & $2.38(0.16-4.00)$ & $<0.001$ \\
\hline NG, present & $14(4 \cdot 4)$ & $1(1.6)$ & $13(5 \cdot 1)$ & 0.233 \\
\hline PUD, present & $17(5 \cdot 3)$ & $3(4.8)$ & $14(5 \cdot 5)$ & 0.843 \\
\hline CAG, present & $223(70.1)$ & $35(56.5)$ & $188(73 \cdot 4)$ & 0.009 \\
\hline MG, present & $29(9.1)$ & $7(11.3)$ & $22(8.6)$ & 0.508 \\
\hline EG, present & $42(19.2)$ & $11(22.6)$ & $31(18.4)$ & 0.240 \\
\hline EE, present & $35(11.0)$ & $11(17 \cdot 7)$ & $24(9.4)$ & 0.059 \\
\hline CSG, present & $30(9.4)$ & $11(17.7)$ & $19(7.4)$ & 0.013 \\
\hline
\end{tabular}

Values are presented as mean $\pm \mathrm{SD}$, number (\%), or median (range).

PG, pepsinogen; OD, optical density; NG, nodular gastritis; PUD, peptic ulcers disease; CAG, chronic atrophic gastritis; MG, metaplastic gastritis; EG, erosive gastritis; EE, erosive esophagitis; CSG, chronic superficial gastritis. 
Table 2. Variables related to the positive stool test findings

\begin{tabular}{|c|c|c|c|c|}
\hline \multirow{2}{*}{ Variable } & \multicolumn{2}{|c|}{ Univariate analysis } & \multicolumn{2}{|c|}{ Multivariate analysis } \\
\hline & OR $(95 \% \mathrm{CI})$ & $p$ value & OR $(95 \% \mathrm{CI})$ & $p$ value \\
\hline Age & $0.959(0.923-0.998)$ & 0.037 & & \\
\hline Sex & $0.724(0.302-1.734)$ & 0.469 & & \\
\hline PG I & $1.049(1.017-1.082)$ & $0.002^{\mathrm{a}}$ & $1.027(1.011-1.043)$ & 0.001 \\
\hline PG II & $0.976(0.885-1.076)$ & 0.624 & & \\
\hline PG I/II & $0.560(0.379-0.830)$ & $0.004^{\mathrm{a}}$ & $0.750(0.635-0.886)$ & 0.001 \\
\hline NG & $0.242(0.020-2.891)$ & 0.262 & & \\
\hline PUD & $0.366(0.039-3.445)$ & 0.380 & & \\
\hline CAG & $0.153(0.032-0.719)$ & 0.017 & & \\
\hline MG & $1.951(0.544-6.999)$ & 0.305 & & \\
\hline EG & $0.495(0.093-2.623)$ & 0.408 & & \\
\hline $\mathrm{EE}$ & $1.119(0.382-3.277)$ & 0.837 & & \\
\hline CSG & $0.453(0.078-2.642)$ & 0.379 & & \\
\hline
\end{tabular}

OR, odds ratio; CI, confidence interval; PG, pepsinogen; NG, nodular gastritis; PUD, peptic ulcers disease; CAG, chronic atrophic gastritis; MG, metaplastic gastritis; EG, erosive gastritis; EE, erosive esophagitis; CSG, chronic superficial gastritis.

${ }^{a}$ After multiple testing corrections, $p$ values of $<0.0042$ were considered significant.

\section{Distribution by endoscopic findings}

The most common endoscopic finding in 318 seropositive subjects was CAG. Eleven subjects showed normal endoscopic finding, whereas 79 had more than two diagnoses that included synchronous CAG and MG. Five of these 79 subjects were diagnosed as having three abnormal endoscopic findings (3 CAG/MG/PUD and 2 CAG/ $\mathrm{MG} / \mathrm{EE})$.

The OD values of the $H$. pylori stool antigen test was significantly higher in the subjects with CAG than those without CAG $(p=0.026)$. On the contrary, it was significantly lower in the subjects with CSG $(p=0.022)$ and those with $\mathrm{EE}(p=0.030)$ (Table 3$)$.

\section{Significant variables related to endoscopic diagnosis}

The mean age of the subjects was oldest in MG (60.3 years) and youngest in NG (43.5 years). Younger subjects had more NG $(p=0.007)$, EE $(p=0.011)$, and CSG $(p=$ o.001), whereas the elderly had more CAG $(p<0.001)$ and MG ( $p<0.001)$. On linear regression analysis (Table 4 ), old age was an independent risk factor for the presence of $M G(p<0.001)$ and the absence of $\mathrm{EE}(p=0.043)$.

The mean serum PG I level was highest in the subjects with PUD, while it was lowest in those with EE. Subjects with EE showed the lowest mean serum PG II level and the highest mean PG I/II ratio. The presence of EE was significantly related to male gender $(p<0.001)$, low serum PG I $(p=0.036)$, and PG II $(p=0.002)$ levels. Furthermore, it was correlated with a higher serum PG I/II ratio $(p=0.040)$. Linear regression analysis revealed that high serum PG I/II ratio $(p=0.040)$ and low serum PG II level $(p=0.004)$ were independent risk factors for the presence of EE.

\section{DISCUSSION}

In this study, the serum PG levels were significantly increased in subjects with a positive stool test than in those with a negative stool test. Significantly increased levels of serum PG I in the subjects with a positive stool test indicated that these subjects had an active $H$. pylo$r i$ infection. Different characteristics of the seropositive subjects according to their stool $\mathrm{H}$. pylori antigen test, serum PG assay, and endoscopic findings imply that a high serum PG I level denotes an ongoing $H$. pylori infection with an increased gastric secreting ability. This further suggests that care should be taken in seropositive subjects with an elevated serum PG I level because of the presence of $H$. pylori in their fecal material.

Serology testing is used widely in East Asia due to its clinical efficacy against $H$. pylori infection [17], and the serum anti-H. pylori IgG titer provides evidence of infection in chronic gastritis subjects even when the gastric 
Table 3. Findings according to the endoscopic diagnosis

\begin{tabular}{|c|c|c|c|}
\hline Variable & Absent & Present & $p$ value \\
\hline NG & 304 & 14 & \\
\hline Age, yr & $51.9 \pm 11.1$ & $43 \cdot 5 \pm 13 \cdot 4$ & 0.007 \\
\hline Male sex & $177(58.2)$ & $5(35 \cdot 7)$ & 0.096 \\
\hline Stool OD & $1.80(0.05-4.00)$ & $2.24(0.13-4.00)$ & 0.312 \\
\hline PG I, ng/mL & $63.4 \pm 28.6$ & $56.2 \pm 19.1$ & 0.436 \\
\hline PG II, ng/mL & $18.9 \pm 11.0$ & $18.6 \pm 8.9$ & 0.935 \\
\hline PG I/II & $4.0 \pm 2.0$ & $3.5 \pm 1.4$ & 0.432 \\
\hline PUD & 301 & 17 & \\
\hline Age, yr & $51.4 \pm 11.4$ & $52.2 \pm 11.2$ & 0.778 \\
\hline Male sex & $170(56.5)$ & $12(70.6)$ & 0.253 \\
\hline Stool OD & $1.81(0.05-4.00)$ & $1.98(0.05-3.55)$ & 0.943 \\
\hline PG I, ng/mL & $62.7 \pm 28.4$ & $68.4 \pm 25.6$ & 0.499 \\
\hline PG II, ng/mL & $18.9 \pm 11.1$ & $17.8 \pm 8.0$ & 0.718 \\
\hline PG I/II & $4.0 \pm 2.0$ & $4.2 \pm 1.2$ & 0.722 \\
\hline CAG & 95 & 223 & \\
\hline Age, yr & $47.9 \pm 11.5$ & $52.3 \pm 11.0$ & $<0.001$ \\
\hline Male sex & $55(57 \cdot 9)$ & $127(57.0)$ & 0.876 \\
\hline Stool OD & $1.27(0.05-4.00)$ & $2.01(0.05-4.00)$ & 0.026 \\
\hline PG I, ng/mL & $57.8 \pm 26.6$ & $65.6 \pm 28.7$ & 0.078 \\
\hline PG II, ng/mL & $17 \cdot 3 \pm 9.9$ & $19.5 \pm 11.2$ & 0.159 \\
\hline PG I/II & $4.0 \pm 2.1$ & $4.0 \pm 1.9$ & 0.919 \\
\hline MG & 289 & 29 & \\
\hline Age, yr & $50.6 \pm 11.1$ & $60.3 \pm 9.5$ & $<0.001$ \\
\hline Male sex & $164(56.7)$ & $18(69.2)$ & 0.581 \\
\hline Stool OD & $1.85(0.05-4.00)$ & $1.38(0.06-3.83)$ & 0.477 \\
\hline PG I, ng/mL & $63.1 \pm 29.0$ & $62.3 \pm 20.8$ & 0.894 \\
\hline PG II, ng/mL & $18.9 \pm 11.2$ & $18.2 \pm 7.7$ & 0.781 \\
\hline PG I/II & $4.0 \pm 2.0$ & $4.0 \pm 1.8$ & 0.999 \\
\hline EG & 276 & 42 & \\
\hline Age, yr & $51.6 \pm 11.6$ & $50.8 \pm 9.8$ & 0.672 \\
\hline Male sex & $156(56.5)$ & $26(61.9)$ & 0.511 \\
\hline Stool OD & $1.9(0.05-4.00)$ & $1.5(0.05-3.91)$ & 0.241 \\
\hline PG I, ng/mL & $63.6 \pm 28.3$ & $58.9 \pm 28.8$ & 0.421 \\
\hline PG II, ng/mL & $19.2 \pm 11.2$ & $16.6 \pm 7.8$ & 0.242 \\
\hline PG I/II & $4.0 \pm 2.0$ & $4.1 \pm 1.9$ & 0.761 \\
\hline $\mathrm{EE}$ & 283 & 35 & \\
\hline Age, yr & $52.1 \pm 11.4$ & $46.9 \pm 9.9$ & 0.011 \\
\hline Male sex & $152(53.7)$ & $30(85.7)$ & $<0.001$ \\
\hline Stool OD & $1.9(0.05-4.00)$ & $0.68(0.06-3.98)$ & 0.030 \\
\hline PG I, ng/mL & $64.4 \pm 29.1$ & $51.6 \pm 16.5$ & 0.036 \\
\hline PG II, ng/mL & $19.7 \pm 11.1$ & $12.3 \pm 6.1$ & 0.002 \\
\hline PG I/II & $3.9 \pm 2.0$ & $4.8 \pm 1.6$ & 0.040 \\
\hline CSG & 288 & 30 & \\
\hline Age, yr & $52.2 \pm 11.3$ & $44.9 \pm 9.9$ & 0.001 \\
\hline
\end{tabular}


Table 3. Continued

\begin{tabular}{lccc}
\hline Variable & Absent & Present & $p$ value \\
\hline Male sex & $162(56.3)$ & $20(66.6)$ & 0.272 \\
Stool OD & $1.91(0.05-4.00)$ & $0.42(0.05-3.83)$ & 0.022 \\
PG I, ng/mL & $64.1 \pm 29.0$ & $52.0 \pm 17.0$ & 0.068 \\
PG II, ng/mL & $19.2 \pm 10.6$ & $15.6 \pm 13.4$ & 0.154 \\
PG I/II & $4.0 \pm 2.0$ & $4.1 \pm 1.7$ & 0.850 \\
\hline
\end{tabular}

Values are presented as mean $\pm \mathrm{SD}$, number (\%), or median (range).

NG, nodular gastritis; OD, optical density; PG, pepsinogen; PUD, peptic ulcers disease; CAG, chronic atrophic gastritis; MG, metaplastic gastritis; EG, erosive gastritis; EE, erosive esophagitis; CSG, chronic superficial gastritis.

Table 4. Linear regression analysis based on the endoscopic findings

\begin{tabular}{|c|c|c|c|c|c|c|c|c|}
\hline \multirow{3}{*}{ Variable } & \multicolumn{8}{|c|}{ Unstandardized coefficient } \\
\hline & \multicolumn{2}{|l|}{ Age } & \multicolumn{2}{|c|}{ PG I } & \multicolumn{2}{|c|}{ PG II } & \multicolumn{2}{|c|}{ PG I/II } \\
\hline & $\mathrm{B}(\mathrm{SE})$ & $p$ value & $\mathrm{B}(\mathrm{SE})$ & $p$ value & $\mathrm{B}(\mathrm{SE})$ & $p$ value & $\mathrm{B}(\mathrm{SE})$ & $p$ value \\
\hline NG & $-7.391(3.749)$ & 0.050 & $-10.757(10.979)$ & 0.328 & $-2.314(4.194)$ & 0.582 & $-0.339(0.765)$ & 0.658 \\
\hline PUD & $-1.074(2.699)$ & 0.691 & $4.626(8.425)$ & 0.583 & $-1.748(3.218)$ & 0.588 & $0.280(0.587)$ & 0.633 \\
\hline CAG & $1.345(2.873)$ & 0.640 & $-2.095(8.184)$ & 0.798 & $-1.537(3.126)$ & 0.623 & $0.201(0.570)$ & 0.725 \\
\hline MG & $8.402(2.143)$ & $<0.001$ & $-3.529(6.613)$ & 0.594 & $-1.308(2.526)$ & 0.605 & $-0.047(0.461)$ & 0.919 \\
\hline EG & $0.609(3.210)$ & 0.850 & $-7.841(9.415)$ & 0.406 & $-3.881(3.597)$ & 0.282 & $0.180(0.656)$ & 0.784 \\
\hline $\mathrm{EE}$ & $-4.016(1.972)$ & 0.043 & $-10.465(6.219)$ & 0.094 & $-6.894(2.376)$ & 0.004 & $0.895(0.433)$ & 0.040 \\
\hline CSG & $-5.013(3.437)$ & 0.146 & $-14.012(10.186)$ & 0.170 & $-4.545(3.891)$ & 0.244 & $0.108(0.710)$ & 0.880 \\
\hline
\end{tabular}

PG, pepsinogen; SE, standard error; NG, nodular gastritis; PUD, peptic ulcers disease; CAG, chronic atrophic gastritis; MG, metaplastic gastritis; EG, erosive gastritis; EE, erosive esophagitis; CSG, chronic superficial gastritis.

biopsy specimens are negative [18]. The stool test has the potential to be useful for the quantitative evaluation of H. pylori status in the stomach [8], but there is a paucity of data on the stool $\mathrm{H}$. pylori antigen test findings according to the serum PG assay and endoscopic findings. Presently, comparison of seropositive subjects with a positive stool test and those with a negative stool test revealed that the stage of $H$. pylori infection was significantly related to the presence of $\mathrm{H}$. pylori in stool. Our study findings add to the previous findings that the presence of $\mathrm{H}$. pylori in stool is linked to an active infection stage as reflected by increased gastric secreting ability. There may be an increased risk of disseminating larger amounts of bacteria via the feces in these seropositive subjects with a positive stool test remain untreated [19].

The serum PG I levels were significantly higher and serum PG I/II ratios were lower in the positive stool group than in the negative stool group in this study. This is due to a greater increase in the serum PG II level than PG I in an infected stomach [20]. PG I is secret- ed only at the fundus while PG II I is secreted throughout the stomach, and therefore, the serum PG II level is known to be more reliable than PG I as a marker for H. pylori-related gastropathies [14,15]. Moreover, a recent study showed that $H$. pylori-related gastric cancers exhibit a higher serum PG II level in conjunction with a shorter allele of the PG II 100 bp ins/del polymorphism [21].

Since serum PG levels increase in the presence of active $H$. pylori infections and decrease in the presence of chronic infections, our study findings indicate that most of the subjects in the stool test negative group had inactive or past infection, and could not actively disseminate the bacterium [19]. This is consistent with previous studies that positivity of the stool antigen test was less accurate in the seropositive subjects with a low serology titer due to the progress of severe gastric atrophy [22]. The present findings are also supported by the findings of a positive correlation between the bacterial density of the gastric mucosa and serum PG I and PG II levels [23]. According to the optimal cut-off values found in this 
study, infected subjects with $H$. pylori in their stool might be reduced in the seropositive subjects with a serum PG I level < 41.5 ng/mL, a PG II level < $8.7 \mathrm{ng} / \mathrm{mL}$, and a PG I/ II ratio $>3.3$. This is due to the less bacterium in the fecal material at chronic infection stage with decreased gastric secreting ability. Their findings are consistent with our study findings that stool antigen test-positive subjects showed a higher prevalence of CAG on endoscopy than the negative subjects, but not the prevalence of gastric corpus atrophy as reflected by the serum PG assay.

Endoscopic findings are useful indicators for $\mathrm{H}$. pylori eradication in seroprevalent populations [24]. Although endoscopic diagnosis was not an independent risk factor for a positive stool test, CAG and NG were more common in the subjects with positive stool test, whereas CSG and EE were more common in those with negative stool test. These findings are consistent with gastric secretability as reflected by the lowest serum PG I and PG II levels and the highest mean PG I/II ratio in the subjects with EE.

The fecal materials were included within 24 hours of sampling to prevent the die-off of $\mathrm{H}$. pylori after prolonged air exposure that might interrupt the survival of $H$. pylori in microaerobic conditions. Nonetheless, there are limitations in this study. First, gastric biopsies were not taken from all subjects in this study. Since we included the subjects who visited for upper gastrointestinal endoscopy for a regular check-up, gastric biopsy was not performed unless there is a suspicious lesion with color and/or structural change. Due to the lack of evaluation based on the updated Sydney system, we could not evaluate the degree of activity, inflammation, gastric atrophy, and intestinal metaplasia according to the stool antigen test findings. Second, frozen stool samples were used for evaluation instead of fresh fecal materials. Third, the results of stool test were expressed as OD values although it has been demonstrated that data expressed as OD are inappropriate for estimating the bacterial load [25]. Despite these limitations, our findings indicate that once the stool test is positive, the subjects exhibit a high serum PG level regardless of the stool test OD values. A relationship between $H$. pylori stool test findings and gastric secreting ability was found depending on the degree of gastric corpus atrophy as reflected by low serum PG I level. Our findings are consistent with previous study demonstrating that $H$. pylori stool test is an useful diagnostic method even with frozen stool samples [26].

In conclusion, there is a link between positive stool $H$. pylori antigen test findings and a serum PG assay in seropositive subjects. Positive stool H. pylori antigen test findings are common in seropositive subjects with increased serum PG I levels indicating an ongoing $H$. pylori infection. Our study findings further suggest that, to reduce the infected subjects with $H$. pylori in their fecal material, seropositive subjects with increased gastric secreting ability need to be treated.

\section{KEY MESSAGE}

1. Most (> 80\%) of the Helicobacter pylori-seropositive subjects showed positive stool test findings.

2. The presence of $\mathrm{H}$. pylori antigen in stool was independently related to a higher serum pepsinogen (PG) I level and a lower serum PG I/II ratio, which denote an active current $H$. pylori infection with increased gastric secreting ability.

3. Seropositive subjects with increased gastric secreting ability tend to have $H$. pylori in their fecal material as reflected by a positive stool $\mathrm{H}$. pylori antigen test finding.

\section{Conflict of interest}

No potential conflict of interest relevant to this article was reported.

\section{Acknowledgments}

This study was supported by the Korean National Research Foundation (2016R1D1A1B02008937 to SunYoung Lee).

\section{REFERENCES}

1. Kokkola A, Rautelin H, Puolakkainen P, et al. Diagnosis of Helicobacter pylori infection in patients with atrophic gastritis: comparison of histology, ${ }_{13} \mathrm{C}$-urea breath test, and serology. Scand J Gastroenterol 2000;35:138-141.

2. Talebkhan Y, Mohammadi M, Rakhshani N, et al. Interobserver variations in histopathological assessment of gastric pathology. Pathology 2009;41:428-432. 
3. Korkmaz H, Kesli R, Karabagli P, Terzi Y. Comparison of the diagnostic accuracy of five different stool antigen tests for the diagnosis of Helicobacter pylori infection. Helicobacter 2013;18:384-391.

4. Dominguez J, Forne M, Blanco S, et al. Comparison of a monoclonal with a polyclonal antibody-based enzyme immunoassay stool test in diagnosing Helicobacter pylori infection before and after eradication therapy. Aliment Pharmacol Ther 2006;23:1735-1740.

5. Shimoyama T, Oyama T, Matsuzaka M, Danjo K, Nakaji S, Fukuda S. Comparison of a stool antigen test and serology for the diagnosis of Helicobacter pylori infection in mass survey. Helicobacter 2009;14:87-90.

6. Choi J, Kim CH, Kim D, et al. Prospective evaluation of a new stool antigen test for the detection of Helicobacter pylori, in comparison with histology, rapid urease test, (13) C-urea breath test, and serology. J Gastroenterol Hepatol 2011;26:1053-1059.

7. Matsuda M, Noda Y, Takemori Y. Utility and limitations of a method for detecting Helicobacter pylori-specific antigens in the stool. J Gastroenterol 2003;38:222-228.

8. Lin HJ, Lo WC, Perng CL, et al. Helicobacter pylori stool antigen test in patients with bleeding peptic ulcers. Helicobacter 2004;9:663-668.

9. Manes G, Balzano A, Iaquinto G, et al. Accuracy of the stool antigen test in the diagnosis of Helicobacter pylori infection before treatment and in patients on omeprazole therapy. Aliment Pharmacol Ther 2001;15:73-79.

10. Miki K, Fujishiro M, Kodashima S, Yahagi N. Longterm results of gastric cancer screening using the serum pepsinogen test method among an asymptomatic middle-aged Japanese population. Dig Endosc 2009;21:78-81.

11. Nardone G, Rocco A, Staibano S, et al. Diagnostic accuracy of the serum profile of gastric mucosa in relation to histological and morphometric diagnosis of atrophy. Aliment Pharmacol Ther 2005;22:1139-1146.

12. Lee SP, Lee SY, Kim JH, et al. Correlation between Helicobacter pylori infection, IgE hypersensitivity, and allergic disease in Korean adults. Helicobacter 2015;20:49-55.

13. Choi HS, Lee SY, Kim JH, et al. Combining the serum pepsinogen level and Helicobacter pylori antibody test for predicting the histology of gastric neoplasm. J Dig Dis 2014;15:293-298.

14. Gritti I, Banfi G, Roi GS. Pepsinogens: physiology, pharmacology pathophysiology and exercise. Pharmacol Res 2000;41:265-281.
15. Lee SY. Endoscopic gastritis, serum pepsinogen assay, and Helicobacter pylori infection. Korean J Intern Med 2016;31:835-844.

16. Lee SY, Moon HW, Hur M, Yun YM. Validation of western Helicobacter pylori IgG antibody assays in Korean adults. J Med Microbiol 2015;64:513-518.

17. Lee SY. Current progress toward eradicating Helicobacter pylori in East Asian countries: differences in the 2013 revised guidelines between China, Japan, and South Korea. World J Gastroenterol 2014;20:1493-1502.

18. Tatemichi M, Sasazuki S, Inoue M, Tsugane S; JPHC Study Group. Clinical significance of IgG antibody titer against Helicobacter pylori. Helicobacter 2009;14:231-236.

19. De Schryver A, Van Winckel M, Cornelis K, Moens G, Devlies G, De Backer G. Helicobacter pylori infection: further evidence for the role of feco-oral transmission. Helicobacter 2006;11:523-528.

20. He CY, Sun LP, Gong YH, Xu Q, Dong NN, Yuan Y. Serum pepsinogen II: a neglected but useful biomarker to differentiate between diseased and normal stomachs. J Gastroenterol Hepatol 2011;26:1039-1046.

21. Kumar S, Kumari N, Mittal RD, Ghoshal UC. Pepsinogen-II $100 \mathrm{bp}$ ins/del gene polymorphism and its elevated circulating levels are associated with gastric cancer, particularly with Helicobacter pylori infection and intestinal metaplasia. Gastric Cancer 2016;19:808-816.

22. Lahner E, Vaira D, Figura N, et al. Role of noninvasive tests (C-urea breath test and stool antigen test) as additional tools in diagnosis of Helicobacter pylori infection in patients with atrophic body gastritis. Helicobacter 2004;9:436-442.

23. Tu H, Sun L, Dong X, et al. Serum anti-Helicobacter pylori immunoglobulin $\mathrm{G}$ titer correlates with grade of histological gastritis, mucosal bacterial density, and levels of serum biomarkers. Scand J Gastroenterol 2014;49:259-266.

24. Hayashi S, Imamura J, Kimura K, Saeki S, Hishima T. Endoscopic features of lymphoid follicles in Helicobacter pylori-associated chronic gastritis. Dig Endosc 2015;27:53-6o.

25. Dore MP, Negrini R, Tadeu V, et al. Novel monoclonal antibody-based Helicobacter pylori stool antigen test. Helicobacter 2004;9:228-232.

26. Yee YK, Yip KT, Que TL, et al. Efficacy of enzyme immunoassay for the detection of Helicobacter pylori antigens in frozen stool specimens: local validation. Aliment Pharmacol Ther 2002;16:1739-1742. 